CNS Spectrums (2016), 21, 43-52. C Cambridge University Press 2013. The online version of this article is published within an Open Access environment subject to the conditions of the Creative Commons Attribution-NonCommercial-ShareAlike licence <http://creativecommons.org/licenses/by-nc-sa/3.0/ > . The written permission of Cambridge University Press must be obtained for commercial re-use. doi: $10.1017 /$ S1092852913000643

\title{
Patient-centered assessment of cognitive symptoms of depression
}

\author{
Sheri E. Fehnel, ${ }^{*}$ Barbara H. Forsyth, ${ }^{\prime}$ Dana B. DiBenedetti, ${ }^{1}$ Natalya Danchenko, ${ }^{2}$ \\ Clément François, ${ }^{3}$ and Thomas Brevig ${ }^{4}$
}

\footnotetext{
${ }^{1}$ Patient-Reported Outcomes, RTI Health Solutions, Research Triangle Park, North Carolina, USA

${ }^{2}$ Health Economics and HTA Management, Lundbeck SAS, Issy-les-Moulineaux, France

${ }^{3}$ Global Outcomes Research, Lundbeck SAS, Issy-les-Moulineaux, France

${ }^{4}$ Corporate Medical Affairs, H. Lundbeck A/S, Valby, Denmark
}

Objective. To identify and explore concepts important to patients with cognitive symptoms of major depressive disorder (MDD) and adapt an existing patient-reported outcome (PRO) measure to assess these symptoms.

Methods. Four focus groups were conducted with MDD patients $(n=33)$ to elicit relevant concepts and determine whether one of several PRO scales could be used to assess cognitive symptoms of depression. Following selection and minor modification of the Perceived Deficits Questionnaire (PDQ), cognitive debriefing interviews were conducted with additional patients $(\mathrm{n}=17)$ to further refine and adapt this measure for use in MDD. Minor revisions based on patient input yielded the PDQ for Depression (PDQ-D).

Results. Focus group participants reported a variety of cognitive symptoms that were classified into 7 general categories: lack of focus and clear thought, memory problems, difficulty with lexical access, difficulty with divided attention, difficulty with decision making, difficulty thinking quickly, and difficulty learning new things. Limitations in work productivity were the most commonly reported impacts of cognitive symptoms. While suggesting a few modifications, focus group participants reacted positively to the PDQ based on the breadth, specificity, and relevance of the items. Cognitive debriefing participants indicated that the modified PDQ items were generally easy to understand and relevant to their experiences with MDD.

Conclusion. Because cognitive symptoms are burdensome to patients with MDD, their assessment is important to optimize treatment outcomes. The PDQ-D has the potential to supplement existing assessment methods, providing unique information important for both comprehensive evaluation of individuals with MDD and evaluation of new treatments.

Received 15 May 2013; Accepted 20 August 2013; First published online 25 September 2013

Key words: Cognition, depression, functioning, measurement, patient-reported outcomes, qualitative, questionnaire.

\section{Introduction}

Depression is a common mental disorder (affecting more than 350 million people of all ages), the leading

\footnotetext{
*Address for correspondence: Sheri E. Fehnel, 200 Park Offices Drive, Research Triangle Park, North Carolina 27709, USA. (Email sfehnel@rti.org)

The authors would like to acknowledge the contributions of Ms. Diana Goss, who managed the project upon which this manuscript is based, as well as those of Ms. Alyssa Dallas, who edited the manuscript. We would also like to thank Dr. Michael Sullivan, developer of the Perceived Deficits Questionnaire, for his permission to adapt and publish this measure. This study was funded by Lundbeck SAS and Takeda Pharmaceuticals.
}

cause of disability worldwide, and a major contributor to the global burden of disease. ${ }^{1}$ Cognitive symptoms are a diagnostic criterion of depression referenced in both the Diagnostic and Statistical Manual of Mental Disorders of the American Psychiatric Association ${ }^{2}$ and in the 10th revision of the World Health Organization's International Statistical Classification of Diseases and Related Health Problems (ICD-10) Classification of Mental and Behavioural Disorders. ${ }^{3}$ In a major depressive episode, cognitive symptoms occur in most patients most of the time, ${ }^{4}$ and often remain even after other symptoms of depression have improved. ${ }^{4-7}$ For example, a recent study estimated that patients with major 
depressive disorder (MDD) in primary care have residual cognitive symptoms $44 \%$ of the time on average between episodes of depression. ${ }^{4}$ Furthermore, cognitive dysfunction persists in patients with MDD who have responded, but not remitted, to treatment with a selective serotonin reuptake inhibitor antidepressant, with as many as $71 \%$ of patients experiencing residual symptoms of decreased concentration/decision making. ${ }^{8}$ Residual symptoms, such as cognitive difficulties, are not only associated with a greater risk of relapse among patients with $\mathrm{MDD},{ }^{9}$ but these symptoms may also be predictive of impaired life function and work productivity. ${ }^{10}$ Improvement in cognitive function is thus an important endpoint of antidepressant treatment, which was also reported by patients with MDD and health care professionals who ranked improvement of cognitive function as the second and fourth priorities (out of 11 outcomes), respectively, in a recent study using the analytic hierarchy process to assess preferences for treatment outcomes. ${ }^{11}$

Despite their prevalence and significant impact on patients' lives, both during and between depressive episodes, cognitive symptoms are neither fully understood nor commonly assessed in clinical research or clinical practice. ${ }^{12}$ Cognitive dysfunction has been demonstrated in patients with MDD relative to age- and gender-matched healthy controls through neuropsychological testing. ${ }^{13-15}$ Although a wide variety of neuropsychological tests are available, such tests are impractical for routine use in clinical practice, because they are time consuming and may require trained professionals for administration. In addition, neuropsychological tests do not address the ways in which cognitive symptoms manifest themselves and affect patient functioning in a real-world setting.

Patient-reported outcome (PRO) measures, typically in the form of a questionnaire, allow for the assessment of cognitive symptoms based on patients' perceptions and experiences in their everyday lives, whereas neuropsychological tests measure performance on isolated cognitive tasks in a controlled environment. PRO measures and neuropsychological tests may thus be administered to assess different aspects of cognitive dysfunction for initial assessment of patients presenting with depression and to monitor treatment outcomes.

Although a PRO measure of cognitive function specifically designed for patients with MDD has not been described in the literature, several existing measures appear to have potential utility in this patient population, including the Perceived Deficits Questionnaire $(\mathrm{PDQ})^{16}$ and the Applied Cognition-General Concerns (ACGC) scale. ${ }^{17}$ While the majority of the cognitive function measures described in the literature focus on symptoms characteristic of dementia, both the PDQ and ACGC were deemed sufficiently generic to be reasonable candidates for use in MDD.
The PDQ was originally developed for use in multiple sclerosis, but has been used to assess cognitive symptoms among patients with other conditions, including MDD. ${ }^{18}$ The PDQ assesses 4 domains of cognitive functioning, which are reflected in the following subscales: attention/ concentration, retrospective memory, prospective memory, and planning/organization. In addition to the full 20item version of the PDQ, a 5-item version is also available for rapid assessment.

The ACGC scale, which is a generic measure intended for use across a wide variety of conditions, is among the tools developed as part of Patient-Reported Outcomes Measurement Information System (PROMIS). PROMIS is an effort funded by the United States' National Institutes of Health to develop banks of items for use in computer adaptive testing, as well as static measures of patient-reported health status. While the full bank contains a total of 34 items, 4-, 6-, and 8-item versions of the ACGC scale are also available. ${ }^{19}$

The objectives of this study were to contribute to the understanding of cognitive symptoms among patients with MDD and to facilitate the future assessment of these symptoms. Specifically, qualitative research was conducted to (1) identify a comprehensive set of cognitive symptoms relevant to patients with MDD, (2) describe the impact of cognitive symptoms on the daily lives of patients with MDD, and (3) adapt an existing PRO measure of cognitive function for use in patients with MDD.

\section{Methods}

The qualitative methods used in this study followed the recommendations pertaining to the modification of existing instruments in the PRO guidance document issued by the United States Food and Drug Administration. ${ }^{20}$ Briefly, to elicit concepts relevant to patients with MDD and to determine if the PDQ or ACGC scale could be adapted for use in this patient population, a series of 4 focus groups was conducted with individuals diagnosed with and being treated for MDD. Following selection and minor modification of the PDQ based on focus group participant feedback, 2 iterative sets of cognitive interviews were conducted to further refine and adapt this PRO measure for use in MDD.

Using semistructured discussion guides developed collaboratively by the authors, the focus groups and cognitive interviews were co-led by the same 2 individuals, each with more than 25 years of qualitative research experience and educational backgrounds in psychology. At the beginning of each session, participants completed a brief background questionnaire and the 20-item Center for Epidemiologic Studies Depression Scale (CES-D) ${ }^{21}$ to facilitate descriptive characterization of the focus group and interview patient samples. All focus 
groups and interviews were audio recorded; qualitative data analysis was conducted using both field notes and transcripts. Additional details pertaining to each step in this process are provided in the following sections.

All study materials and procedures were reviewed and approved by RTI International's Institutional Review Board before patient recruitment was initiated. Written informed consent was obtained from all patients prior to their participation in this research study.

\section{Focus groups}

Four focus groups were conducted with 33 adults diagnosed with MDD. All participants had received a primary diagnosis of MDD at least 3 months prior to screening, were currently taking an antidepressant, and reported 2 or more cognitive symptoms during screening when prompted with a short list of potential symptoms. Focus group participants were referred by treating general practitioners and psychiatrists; additional screening criteria eliminated patients with comorbid conditions or those taking medications likely to cause cognitive symptoms.

Two of the focus groups consisted of younger patients with MDD (18-45 years), and 2 groups included patients with MDD across a broader age range (18-65 years). The rationale for conducting several groups with younger patients was to determine if there were systematic differences in the types or attribution of cognitive symptoms reported between this cohort and the more general sample. In particular, it was anticipated that younger participants would be less likely to attribute cognitive symptoms to aging. An additional criterion was also imposed for the focus groups with younger MDD patients with the intention of further minimizing the potential for cognitive symptoms unrelated to MDD; specifically, female patients experiencing symptoms of menopause (eg, hot flashes) were excluded from these two groups. Two of the focus groups (one with each age group) were held in Raleigh, North Carolina, in April 2011, while the other two were conducted in San Antonio, Texas, in May 2011.

Following participants' completion of the CES-D, the 2 moderators posed open-ended questions to facilitate discussion of patients' MDD symptoms, eventually focusing on their cognitive symptoms. First, moderators asked patients to freely recall the "major symptoms" they associated with their MDD. Once free recall elicited no new information about cognitive symptoms, moderators prompted additional recall either by asking for more detail about previously reported cognitive symptoms or by asking how MDD affects patients' work or other daily activities. Once prompted recall generated no new information about cognitive symptoms, moderators probed for experiences with particular cognitive symptoms, including trouble with focusing or concentrating, thinking clearly, finding words, remembering things, learning new information, expressing oneself clearly, planning, keeping organized, and making decisions. This three-pronged question-asking strategy (ie, free recall, prompting, and probing) was used to gain information not only about the cognitive symptoms that patients experienced, but also about the relative salience of the cognitive symptoms reported. Additional discussion topics included patients' perceptions of the relations between cognitive symptoms and depression, the relative importance of reported cognitive symptoms, effects of treatment on cognitive symptoms, and the impacts of patients' cognitive symptoms on their daily lives and activities.

Following concept elicitation and discussion, participants provided feedback on the full (20-item) PDQ and the 8-item version of the ACGC scale to determine if one of these PRO measures might be appropriate for adaptation and use in MDD. The moderators counterbalanced the order for presenting and discussing the 2 scales to control for potential order effects on patients' responses and reactions.

\section{Preliminary modification of the PDQ}

The PDQ was selected for further testing and adaptation for use in MDD, based on a clear consensus among participants in all 4 focus groups that its items were more comprehensive and relevant to their experiences with depression. Based on feedback from the focus group participants, minor modifications were made to the PDQ, with permission from the developer, prior to the cognitive interviews. Specifically, the 4-week reference period was shortened to 7 days, and the response category descriptions were expanded.

\section{Cognitive interviews}

To further refine and adapt the PDQ for use in depression, 2 iterative sets of cognitive interviews were conducted with a total of 17 additional patients with MDD. Although the cognitive debriefing participants were required to meet the same screening criteria as the focus group participants, these patients were selfreferred rather than clinician-referred. Recruitment goals were set such that roughly half of the participants were selected from a younger age cohort (18-44 years) and half were selected from an older age cohort (45-65 years). The 2 sets of interviews were conducted in Bethesda, Maryland, and Raleigh, North Carolina, in October and November of 2011, respectively.

Following participants' completion of the CES-D, interviewers posed a series of open-ended questions to identify participants' cognitive symptoms and related 
experiences. This concept elicitation exercise was conducted prior to the initiation of cognitive debriefing to avoid bias and to encourage participants to describe their cognitive symptoms in their own words.

The interviews then focused on a cognitive debriefing review of the draft PDQ for Depression (PDQ-D) to gain insight into participants' interpretations of individual questionnaire items, participants' strategies for answering each item, and the relevance of each item to participants" experiences. A "think-aloud" technique was used, wherein the interviewer asked participants to read individual items and describe, in their own words, things participants thought about as they interpreted and selected a response for each question. Interviewers also asked scripted probe questions to get specific information about individual items if participants' think-aloud responses did not touch on important aspects of the items. Finally, interviewers asked whether the questionnaire missed any cognitive symptoms that participants associated with their depression.

\section{Results}

\section{Participants}

As summarized in Table 1, the MDD patients who participated in the focus groups $(\mathrm{n}=33)$ and cognitive debriefing interviews $(n=17)$ were clinically and demographically similar, with the exception of their level of education. Specifically, the cognitive interview participants, on average, were more highly educated than the focus group participants. This difference may be due to the differing recruitment strategies and suggests that patients with higher levels of education may be more likely to self-refer for participation in qualitative research. The consistency of concepts identified across these patient samples, however, supports the content validity of the PDQ-D for use across the MDD patient population (ie, regardless of the level of patient education). At the time of their participation in this study, $10(20 \%)$ of the patients had a CES-D score $<16$, the threshold typically considered indicative of clinical depression. This finding was not surprising, given that all of these patients were under treatment at the time of the study and most had responded, at least partially, to therapy. While the inclusion of patients with more severe depression was initially targeted, physicians did not feel comfortable referring patients with severe symptoms for participation. It is also unlikely that acutely depressed patients would have participated fully in the discussions or been as capable of describing changes in their cognitive symptoms as those with lesser levels of symptomatology at the time of the study.

\section{Focus groups}

\section{Cognitive symptom reports}

Focus group participants attributed a wide variety of cognitive symptoms to their depression. To facilitate summarization and interpretation, these symptoms were classified into 7 general categories, comprising (1) lack of focus and clear thought, (2) memory problems, (3) difficulty with lexical access, (4) difficulty with divided attention, (5) difficulty with decision making, (6) difficulty thinking quickly, and (7) difficulty learning new things. The numbers of focus groups reporting specific symptoms within each of these categories are shown in Table 2. To demonstrate the relative importance and salience among these symptoms, the numbers of focus groups reporting these symptoms are provided separately for free recall,

TABLE 1. Characteristics of focus group and cognitive interview participants

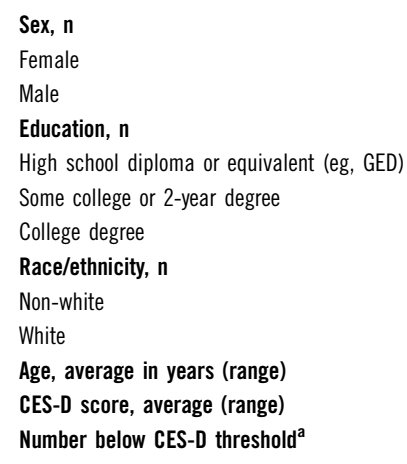

$\begin{array}{cc}24 & 12 \\ 9 & 5 \\ 9 & \\ 14 & 0 \\ 10 & 4 \\ & 13 \\ 15 & \\ 18 & 7 \\ 45.2(23-65) & 10 \\ 25.5(6-53) & 42.6(28-60) \\ 7 \text { patients }<16 & 29.6(10-48) \\ & 3 \text { patients }<16\end{array}$

CES-D = Center for Epidemiologic Studies Depression Scale; GED = general education development.

${ }^{a}$ CES-D scores $\geq 16$ are generally recognized as indicative of depressive illness. 


\begin{tabular}{|c|c|c|c|c|}
\hline \multirow[b]{3}{*}{ Patient-reported symptom } & \multirow[b]{3}{*}{ Total } & \multicolumn{3}{|c|}{$\begin{array}{l}\text { Number of focus groups where at least } 1 \text { patient } \\
\text { reported symptom (Total number of groups }=4 \text { ) }\end{array}$} \\
\hline & & \multicolumn{3}{|c|}{ Type of recall } \\
\hline & & Free & Prompted & Probec \\
\hline \multicolumn{5}{|l|}{ Lack of focus or clear thought } \\
\hline Difficulty focusing attention; loss of concentration; short attention span & 4 & 1 & 3 & 0 \\
\hline Trouble thinking clearly; losing train of thought & 4 & 0 & 2 & 2 \\
\hline Difficulty getting and staying organized & 4 & 0 & 1 & 3 \\
\hline Distractible & 2 & 0 & 2 & 0 \\
\hline Disconnected thoughts; mind jumps from one thing to another & 2 & 0 & 2 & 0 \\
\hline Lack of attention to ongoing activity; 'zoning off'; daydreaming & 2 & 0 & 1 & 1 \\
\hline \multicolumn{5}{|l|}{ Memory problems } \\
\hline Forgetting where things are; difficulty keeping track of objects (eg, lost keys) & 4 & 1 & 2 & 1 \\
\hline Forgetting activity-related details (eg, purchases made, whether activities completed) & 3 & 0 & 3 & 0 \\
\hline Forgetting, missing, or confusing appointments & 2 & 1 & 0 & 1 \\
\hline Forgetting intent (eg, walk into room and forget why) & 2 & 0 & 1 & 1 \\
\hline $\begin{array}{l}\text { Short- and long-term memory problems; general forgetfulness (eg, birthdays, names) } \\
\text { Difficulty with lexical access }\end{array}$ & 2 & 0 & 2 & 0 \\
\hline $\begin{array}{l}\text { Trouble retrieving specific words; trouble finding the right word } \\
\text { Difficulty with divided attention }\end{array}$ & 3 & 0 & 1 & 2 \\
\hline Difficulty multitasking & 2 & 0 & 2 & 0 \\
\hline Difficulty with switching from one task to another; difficulty juggling tasks & 2 & 0 & 2 & 0 \\
\hline Difficulty dealing with multiple pieces of information at once & 2 & 0 & 1 & 1 \\
\hline Difficulty making decisions & 3 & 0 & 0 & 3 \\
\hline Difficulty learning new things & 2 & 0 & 1 & 1 \\
\hline
\end{tabular}

Symptoms reported by only 1 participant (each) included 'foggy or hazy thought,' 'mind goes blank,' and 'absent-minded.'

prompted recall, and probed recall. Although older participants were somewhat more likely to mention potentially confounding sources of cognitive symptoms, such as age and menopausal symptoms, the results were generally similar across the 4 focus groups and are, therefore, described together.

Most of the symptoms that patients freely recalled were not in the cognitive domain, including lack of motivation, fatigue, general disinterest, and depressed mood. Spontaneous reports of cognitive symptoms were very rare, suggesting that few cognitive symptoms were salient or prominent enough to be reported without prompting. Only concentration and memory problems were reported in this free recall context, and each symptom was reported spontaneously within only 1 of the 4 focus groups. Many more cognitive symptoms were reported when patients were prompted to consider this type of symptom or were specifically queried (or probed) about particular symptoms. The cognitive symptoms that patients identified as the most important were difficulties with concentration or focused attention and memory; other prominent cognitive symptoms included trouble thinking clearly and disorganization.

While all focus group participants reported experiencing cognitive symptoms when prompted, there are several factors that may have contributed to the rarity of spontaneous reports. In particular, many patients were uncertain about the relation between their ongoing cognitive symptoms and typical fluctuations in the severity of their depression, while others noted confounding factors such as age and menopause that made it difficult to confidently attribute their cognitive symptoms to depression. In addition, some patients seemed to have trouble making clear distinctions between the cognitive and other symptoms of depression and noted that their cognitive symptoms amplified the other symptoms. For example, difficulties concentrating or focusing attention contributed to increased anxiety, decreased motivation, and fatigue. Patients also noted that their noncognitive symptoms could influence their cognitive symptoms. For example, both low motivation and fatigue could limit attention to detail and contribute to recall problems.

While most patients in the focus groups reported impacts that they attributed to cognitive symptoms, they reported many more impacts that they attributed to other symptoms of MDD, including general disinterest, anxiety, lack of motivation, and preoccupied thoughts. In other words, impacts from the noncognitive symptoms were generally more salient and significant than those attributed to cognitive symptoms. In addition, participants commonly reported the belief that many of their cognitive symptoms were either caused by or made 
worse by other symptoms of MDD. For example, many of the cognitive symptoms were influenced by negative thoughts with which patients were preoccupied, as well as problems with motivation and/or fatigue. Because noncognitive symptoms were intrinsically linked to the cognitive symptoms patients experienced, it was difficult for most focus group participants to report impacts that were purely cognitive.

Several patients reported that their cognitive symptoms affected their productivity at work. Impacts on productivity at work most commonly included difficulties meeting deadlines, decreased quality in work products due to inattention to task details, and failure to complete assigned tasks and/or failure to follow through on assigned responsibilities. These impacts were largely associated with difficulties in concentration or focused attention and memory.

In comparison to issues with productivity, fewer patients reported that cognitive symptoms affected family relationships or social activities. Home and family impacts included strained relationships with family members and difficulties meeting family responsibilities, typically due to forgetfulness. Some patients mentioned that problems with decision making sometimes made it more difficult to make plans with friends.

The focus group participants generally indicated that their cognitive symptoms were most severe when they were most actively depressed and finding it difficult to function in any capacity. For example, patients in several groups talked about periods in which they had been so severely depressed that they were unable to think about much of anything other than the circumstances contributing to their depression and how they were feeling at the time. Some patients could also see a clear link between fluctuations in the severity of their depression and the presence or severity of cognitive symptoms they experienced on a more regular basis, most commonly noting difficulties with focus and attention. In addition, while some patients reported improvements in their memory and other cognitive symptoms following treatment for MDD, others indicated that their antidepressants seemed to have no effect on these symptoms.

\section{PRO questionnaire review}

Participants' initial reactions to the ACGC scale differed depending on whether they reviewed this measure before or after they reviewed the PDQ. In particular, most patients in the 2 groups that reviewed the ACGC scale first were initially positive in their feedback regarding this instrument; whereas patients in the 2 groups that reviewed the ACGC scale after the PDQ were generally more critical of this measure from the beginning. Irrespective of the presentation order, the vast majority of patients ultimately indicated that the 8-item ACGC scale would not be a particularly effective measure of the cognitive symptoms they experienced, particularly in comparison to the 20-item PDQ. Also, several patients observed that it was difficult to make comparisons to how they "usually" felt as required in some of the ACGC-scale items, because their depression, their cognitive symptoms, and their treatment were all long-standing.

Regardless of whether they reviewed the PDQ first or second, the vast majority of focus group participants (across all 4 groups) had very positive reactions to and expressed a clear preference for this measure, compared to the ACGC scale. The specificity of the PDQ items was a major factor in this preference. Patients found it easier to respond to the PDQ considering the particular tasks queried in the PDQ and more difficult to respond in relation to the general capabilities queried in the ACGC scale. In particular, patients noted that the PDQ items provided more detail and were not as generic as those on the ACGC scale. Also, patients thought that the PDQ covered a broader range of concepts, including the cognitive symptoms they had experienced.

While patients preferred the items included in the PDQ, they also liked the response scale used in the ACGC instrument, because it provided specific definitions for the frequency terms that label the response points. For example, according to the ACGC response scale, "sometimes" should be interpreted as " 2 or 3 times in the past 7 days." Most patients thought the PDQ would be more effective and easier to answer if it offered similar definitions for the PDQ response scale labels.

Patients also commented on the difference in the recall periods of the 2 scales. While some patients preferred the PDQ's 4-week recall period, which allowed them to convey "patterns" of experiences cumulated over time, others preferred the 7-day recall period used in the ACGC scale, which facilitated recall. Most pertinent to the utility of the measure, preferences for the 7-day recall period were primarily driven by patients' concerns that memory problems associated with MDD could make it difficult to report over a longer time frame.

Based on these findings, more detailed definitions were added to the original PDQ response categories (eg, from "rarely" to "rarely [once a week]," from "often" to "often [about once a day]"). and the recall period was shortened from 4 weeks to 7 days prior to testing in the cognitive interviews.

\section{Cognitive interviews}

\section{Cognitive symptom reports}

In response to a general question about their depressionrelated symptoms, 6 of the 17 cognitive interview 
TABLE 3. Examples of minor changes made to the draft PDQ-D based on cognitive interview results

\begin{tabular}{lcc}
\hline Draft PDQ-D question wording & Revised PDQ-D question wording & Reason for revision \\
\hline $\begin{array}{c}\text { During the past } 7 \text { days, how often did you miss } \\
\text { appointments and meetings you had scheduled? }\end{array}$ & $\begin{array}{c}\text { During the past } 7 \text { days, how often did you forget } \\
\text { appointments and meetings you had scheduled? }\end{array}$ & $\begin{array}{c}\text { Patients thought 'miss appointments' had a noncognitive, } \\
\text { motivational component. } \\
\text { When tested, the revised item helped patients focus on } \\
\text { memory issues. }\end{array}$ \\
$\begin{array}{c}\text { During the past } 7 \text { days, how often did you have } \\
\text { trouble concentrating on things like watching a } \\
\text { television program or reading a book? }\end{array}$ & $\begin{array}{c}\text { During the past } 7 \text { days, how often did you have trouble } \\
\text { concentrating on what you were reading? }\end{array}$ & $\begin{array}{c}\text { Patients had difficulty selecting a single response because } \\
\text { of different experiences watching television and reading } \\
\text { a book. }\end{array}$ \\
& & $\begin{array}{c}\text { When tested, the revised item was easy for patients to } \\
\text { answer and encouraged them to think about reading at } \\
\text { work as well as leisure reading. }\end{array}$
\end{tabular}

participants mentioned 1 or more cognitive symptoms: 3 participants reported a focus on negative thoughts; 3 participants reported difficulties with concentration, focus, or clear thinking; 1 participant reported problems making decisions; 1 participant reported difficulties keeping organized; and 1 participant reported memory issues.

When interviewers explicitly asked whether participants noticed depression affecting memory, concentration, or the ability to think clearly, 16 of the 17 participants mentioned at least 1 cognitive symptom. In response to the more specific question about cognitive symptoms, the most frequently mentioned cognitive symptoms were difficulties with memory and difficulties with concentration. Less frequently mentioned were difficulties with attention, difficulties multitasking, difficulties thinking clearly, difficulties planning or prioritizing, difficulties making decisions, issues with lexical access, issues with learning, and a focus on negative thoughts. Although 1 participant did not initially report cognitive symptoms, subsequent debriefing responses suggested that this participant also experienced depression-related cognitive symptoms.

Cognitive interview participants were not explicitly asked to describe the impact of their cognitive symptoms. Nonetheless, several participants spontaneously reported impact on effectiveness at work and on organization and relations at home. Thus, the interview results reported here are in accord with earlier focus group results and support the notion that while cognitive symptoms are prevalent and impactful, they are not as salient or as likely to be reported spontaneously as other symptoms of MDD.

\section{Review of the draft $P D Q-D$}

Although some item revisions were recommended in the first set of interviews and subsequently tested in the second set of interviews, participants generally reported that the PDQ-D items were easy to understand and answer, as well as important and relevant to their experiences. Revisions to the draft PDQ-D based on MDD patient input included minor clarifications to the wording of the instructions, 6 questions, and the response options.

Overall, participants indicated that the items were appropriate for weekly assessment and concurred that response categories defined by a combination of verbal and numerical frequency indicators were most effective and easiest to use. A few examples of clarifications in question wordings are included in Table 3 . The final version of the PDQ-D, with reference to the original PDQ where changes were made, is included as Supplementary material.

While age comparisons were not central to the research conducted here, the cognitive interview design recruited roughly equal numbers of participants from a younger (18-44 years) and an older (45-65 years) age cohort, providing an opportunity to explore participants' opinions about item responses that might be affected by age either in addition to or instead of depression. While 3 of the 17 participants noted that their age might be a factor in the memory-related issues they experienced, only 1 identified age as a possible factor in responding to a specific item.

It may also be interesting to note that participants' debriefing comments suggest that from the patients' perspectives, some of the PDQ-D items may be multidimensional. For example, participants observed that some kinds of forgetting are likely to happen when they are distracted or multitasking. Thus, some of the memory items may address both memory and concentration.

\section{Discussion}

Although cognitive symptoms may not be the main reason that patients seek treatment during a major depressive episode, they may exacerbate other symptoms and tend to persist even after a patient's mood has improved. Despite their prevalence and impact, patients may not spontaneously report cognitive symptoms to their physicians. During the acute phase of a depressive episode, cognitive symptoms are likely to be overshadowed by mood-related symptoms. Following the 
acute phase, patients may feel that cognitive symptoms are not attributable to their depression but to other factors, such as age, menopause, lack of sleep, or medication side effects. Patients may also have trouble making clear distinctions between cognitive symptoms and other issues such as low motivation and fatigue. Because patients may not spontaneously report cognitive symptoms, clinicians need to prompt patients to describe any cognitive symptoms they may be experiencing.

While cognitive symptoms are not a primary target of initial treatment for physicians, ${ }^{22}$ it is important to explore patients' experiences related to cognitive symptoms in order to plan for treatment success after stabilization of mood and interest. Although physicians may consider patients to be in remission and deem a treatment successful at this point, residual symptoms may still be bothersome to patients. ${ }^{23,24}$ Where appropriate, treatment plans that address cognitive and other residual symptoms should be developed to optimize long-term patient outcomes. A brief, patient-reported assessment of cognitive symptoms that patients associate with depression, such as the PDQ-D, could offer a standard and efficient approach to facilitate both identification of cognitive symptoms and progress toward treatment goals.

In patients with multiple sclerosis (MS), for which the PDQ was originally developed, self-perceived cognitive dysfunction reported by patients using different measures does not generally correlate well with results of neuropsychological tests, ${ }^{25-27}$ suggesting that the PDQ is complementary, rather than an alternative, to neuropsychological testing in MS patients. Self-reported cognitive dysfunction may correlate with depression symptoms in MS patients, ${ }^{28}$ but cognitive complaints should not be discounted due to depression, because cognitive dysfunction reported using the PDQ has been demonstrated to reflect subtle declines in immediate memory and processing speed index scores independent of mood, fatigue, and physical impairment. ${ }^{29}$

In a recent study in patients with MDD, higher scores on the original PDQ (indicating greater cognitive symptom severity as perceived by patients) were associated with poorer performance on a series of objective cognitive tests, including the Hopkins Verbal Learning Test-Revised, Digit Span Forward, Digit Span Backward, and D-KEFS-Letter Fluency Test (Dr. Jennifer C. Samp, PharmD, MS, written communication, August 2013). Among patients with MDD, the association with the PDQ-D would likely be stronger given the greater relevance of the item content to this patient population. Given the time requirements and logistical difficulties associated with neuropsychological assessment in clinical practice, the PDQ-D may, at a minimum, help clinicians to identify patients with significant cognitive dysfunction and target treatments accordingly. A PRO measure such as the PDQ-D can also provide clinicians with information that cannot be gleaned from performance tests. Specifically, the PDQ-D has the potential to provide succinct and interpretable information regarding the status and impact of patients' cognitive symptoms on their everyday lives based on their own perceptions and experiences.

Although the present study generally supports the utility of the PDQ-D across the MDD patient population, it does have a few weaknesses that require further investigation. For example, it may be valuable to conduct additional cognitive debriefing interviews with patients who have no more than a high school education to fully demonstrate that such individuals can easily comprehend and answer the questionnaire items. In addition, it is difficult to assess the practical importance of participants' observations about potential relations among age, memory, and depression, because relatively few participants commented on this topic, and such observations may be unduly influenced by expectations. A quantitative evaluation, perhaps in the context of an observational study, would provide a stronger and more rigorous basis for assessing the potential impact of age on cognitive symptoms and depression. Similarly, study participants noted that some kinds of forgetting are likely to happen when they are distracted or multitasking. Thus, some of the PDQ-D memory items may address both memory and concentration. While these debriefing comments are suggestive, psychometric evaluation would provide a stronger and more rigorous basis for identifying multidimensional items and validating appropriate scoring algorithms.

In addition to further exploring the impact of age on cognitive symptoms and item dimensionality, a quantitative evaluation is currently being conducted to determine whether the PDQ-D items yield reliable results, whether the items support the original subscale structure, and whether item reduction will enhance the effectiveness, sensitivity, and specificity of the PDQ-D. The results will also demonstrate the degree of correlation between the PDQ-D and measures of depression severity and general functioning, as well as other assessments of cognition. It is anticipated that this evaluation will further support the utility of the PDQ-D, demonstrate its psychometric properties, and facilitate the interpretation of scores.

Finally, the development of a short-form version is also planned that will facilitate screening and maximize efficiency of assessment in the clinical setting. Because the content addressed in the PDQ-D does not represent a significant shift from that in the original PDQ, it is possible that the PDQ-5, with appropriate modification (eg, change in response scale to match the PDQ-D), would be appropriate for use in depression. However, the optimal number and combination of items from the PDQ-D for the purpose of rapid assessment will need to be determined empirically. 


\section{Conclusion}

Although spontaneous reports of cognitive symptoms were rare in the focus groups, many cognitive symptoms were reported when the MDD patients were prompted to consider this type of symptom or were specifically probed about particular symptoms. Patients generally reported cognitive symptoms as burdensome and impactful, making assessment of these symptoms important to optimize treatment outcomes. Patients reacted more positively to the PDQ than to the ACGC scale based on the breadth, specificity, and relevance of the PDQ items. The PDQ was modified based on input from the MDD patients participating in both the focus groups and cognitive debriefing interviews. The resulting PDQ-D was generally found to be easy to understand and relevant to patients' experiences with cognitive symptoms of MDD. While further quantitative evaluation is warranted, the results of this qualitative study suggest that the PDQ-D has the potential to supplement existing assessment methods, and provide unique information important for comprehensive evaluation of patients with MDD.

\section{Disclosures}

Thomas Brevig has the following disclosure: H. Lundbeck, employee, salary. Sheri Fehnel has the following disclosure: Lundbeck SAS, consultant, research support. Barbara Forsyth has the following disclosure: Lundbeck SAS, independent contractor, research support. Dana DiBenedetti has the following disclosure: Lundbeck SAS, consultant, manuscript development support. Natalya Danchenko has the following disclosure: Lundbeck SAS, employee, salary. Clément François has the following disclosure: Lundbeck SAS, employee (Director), salary.

\section{Supplementary material}

To view the full 20-item PDQ-D, please visit http:// dx.doi.org/10.1017/S1092852913000643

\section{REFERENCES:}

1. World Health Organization. Fact Sheet No. 369. October 2012. http://www.who.int/mediacentre/factsheets/fs369/en/index.html. Accessed March 3, 2013.

2. American Psychiatric Association. Diagnostic and Statistical Manual of Mental Disorders, 4th ed., text revision. Washington, DC: American Psychiatric Association; 2000.

3. World Health Organization. The ICD-10 Classification of Mental and Behavioural Disorders: Clinical Descriptions and Diagnostic Guidelines. Geneva: World Health Organization; 1992.

4. Conradi HJ, Ormel J, de Jonge P. Presence of individual (residual) symptoms during depressive episodes and periods of remission: a 3-year prospective study. Psychol Med. 2011; 41(6): 1165-1174.

5. Baune BT, Miller R, McAfoose J, et al. The role of cognitive impairment in general functioning in major depression. Psychiatry Res. 2010; 176(2-3): 183-189.
6. Pedrelli P, Baer L, Losifescu DV, Fava M. Relationship between residual symptoms of depression and self-reported cognitive impairment. CNS Spectr. 2010; 15(1): 46-51.

7. Fava M, Graves LM, Banazzi F, et al. A cross-sectional study of the prevalence of cognitive and physical symptoms during long-term antidepressant treatment. J Clin Psychiatry. 2006; 67(11): 1754-1759.

8. McClintock SM, Husain MM, Wisniewski SR, et al. Residual symptoms in depressed outpatients who respond by $50 \%$ but do not remit to antidepressant medication. J Clin Psychopharmacol. 2011. 31(2): 180-186.

9. Judd LL, Akiskal HS, Maser JD, et al. Major depressive disorder: a prospective study of residual subthreshold depressive symptoms as predictor of rapid relapse. J Affect Disord. 1998; 50(2-3): $97-108$.

10. Jaeger J, Berns S, Uzelac S, Davis-Conway S. Neurocognitive deficits and disability in major depressive disorder. Psychiatry Res. 2006; 145(1): 39-48.

11. Danner M, Hummel JM, Volz F, et al. Integrating patients' views into health technology assessment: analytical hierarchy process (AHP) as a method to elicit patient preferences. Int J Technol Assess Health Care. 2011; 27(4): 369-375.

12. Greer TL, Kurian BT, Trivedi MH. Defining and measuring functional recovery from depression. CNS Drugs. 2010; 24(4): 267-284.

13. Douglas KM, Porter RJ. Longitudinal assessment of neuropsychological function in major depression. Aust $N Z \mathrm{~J}$ Psychiatry. 2009; 43(12): 1105-1117.

14. Iverson GL, Brooks BL, Young AH. Identifying neurocognitive impairment in depression using computerized testing. Appl Neuropsychol. 2009; 16(4): 254-261.

15. Gualtieri CT, Johnson LG, Benedict KB. Neurocognition in depression: patients on and off medication versus healthy comparison subjects. J Neuropsychiatry Clin Neurosci. 2006; 18(2): 217-225.

16. Sullivan MJL, Edgley K, Dehoux E. A survey of multiple sclerosis, part 1: perceived cognitive problems and compensatory strategy use. Canadian Journal of Rehabilitation. 1990; 4: 99-105.

17. Gershon R, Rothrock NE, Hanrahan RT, et al. The development of a clinical outcomes survey research application: assessment center. Qual Life Res. 2010; 19(5): 677-685.

18. Lawrence C, Roy A, Harikrishnan V, Yu S, Dabbous OH. The association between self-perceived cognitive difficulties and level of depression among employees with current depression. Podium presentation at the16th Annual International Meeting of the International Society for Pharmacoeconomics and Outcomes Research (ISPOR). May 21-25, 2011 in Baltimore, MD, USA. Value Health. 2011;14(3 Suppl):A3.

19. National Institutes of Health. PROMIS Instruments Available for Use. February 2011. http://www.nihpromis.org/Documents/ Item_Bank_Tables_Feb_2011.pdf. Accessed February 10, 2013.

20. U.S. Department of Health and Human Services (DHHS). Food and Drug Administration. Guidance for industry patient-reported outcome measures: use in medical product development to support labeling claims. December 2009. http://www.fda.gov/downloads/ Drugs/GuidanceComplianceRegulatoryInformation/Guidances/ UCM193282.pdf. Accessed February 10, 2013.

21. Radloff LS, Locke BZ. Center for Epidemiologic Studies Depression Scale. In: Rush AJ, ed. Handbook of Psychiatric Measures. Washington, DC: American Psychiatric Association; 2000: 523-525.

22. Demyttenaere K, Ansseau M, Constant E, et al. Do general practitioners and psychiatrists agree about defining cure from depression? The DEsCRIBE ${ }^{\mathrm{TM}}$ survey. BMC Psychiatry. 2011; 11: 169 . 
23. Zimmerman M. Discordance between researchers and patients in defining remission from depression. J Clin Psychiatry. 2012; 73(9): 1262-1263.

24. Zimmerman M, Martinez JH, Attiullah N, et al. Symptom differences between depressed outpatients who are in remission according to the Hamilton Depression Rating Scale who do and do not consider themselves to be in remission. J Affect Disord. 2012; 142(1-3): 77-81.

25. Benedict RH, Munschauer F, Linn R, et al. Screening for multiple sclerosis cognitive impairment using a selfadministered 15-item questionnaire. Mult Scler. 2003; 9(1): 95-101.
26. Taylor R. Relationships between cognitive test performance and everyday cognitive difficulties in multiple sclerosis. Br J Clin Psychol. 1990; 29(pt 2): 251-253.

27. Beatty WW, Monson N. Metamemory in multiple sclerosis. J Clin Exp Neuropsychol. 1991; 13(2): 309-327.

28. Lovera J, Bagert B, Smoot KH, et al. Correlations of Perceived Deficits Questionnaire of Multiple Sclerosis Quality of Life Inventory with Beck Depression Inventory and neuropsychological tests. J Rehabil Res Dev. 2006; 43(1): 73-82.

29. Marrie RA, Chelune GJ, Miller DM, Cohen JA. Subjective cognitive complaints relate to mild impairment of cognition in multiple sclerosis. Mult Scler. 2005; 11(1): 69-75. 